\title{
Sur la baisse récente de la population carcérale en Allemagne. Éléments de mise en perspective
}

\section{Grégory Salle et Barbara Bauduin}

\section{(2) OpenEdition}

1 Journals

Édition électronique

URL : http://journals.openedition.org/ifha/8073

DOI : $10.4000 /$ ifha. 8073

ISSN : 2198-8943

\section{Éditeur}

IFRA - Institut franco-allemand (sciences historiques et sociales)

\section{Édition imprimée}

Date de publication : 31 décembre 2014

ISSN : 2190-0078

\section{Référence électronique}

Grégory Salle et Barbara Bauduin, «Sur la baisse récente de la population carcérale en Allemagne. Éléments de mise en perspective », Revue de l'IFHA [En ligne], 6 | 2014, mis en ligne le 31 décembre 2014, consulté le 10 décembre 2020. URL : http://journals.openedition.org/ifha/8073 ; DOI : https:// doi.org/10.4000/ifha.8073

Ce document a été généré automatiquement le 10 décembre 2020.

(CIFHA 


\title{
Sur la baisse récente de la population carcérale en Allemagne. Éléments de mise en perspective
}

\author{
Grégory Salle et Barbara Bauduin
}

\section{Introduction : les prisons allemandes en berne?}

1 «En Allemagne, on ferme des prisons faute de détenus. $»^{1}$ Le titre de cet article publié par le site d'information Slate en janvier dernier ne pouvait manquer d'accrocher le regard au moment où, en France, le nombre de détenus battait un nouveau record. Après avoir passé de longues années au coude à coude avec son voisin allemand dans le ventre mou des classements internationaux, l'Hexagone y faisait subitement figure de contre-modèle. S'appuyant sur deux articles publiés par Die Welt et la Süddeutsche Zeitung, l'auteur faisait part d'une chute de la démographie carcérale outre-Rhin avoisinant, d'après les chiffres donnés pour 2007 et 2013, les $22 \%$ en six ans. En mars, c'était au tour d'Arte de diffuser un reportage consacré au même cas de fermeture d'établissements et hardiment intitulé : «Allemagne : la fin des prisons $~^{2}$. Au-delà ou en-deçà de cette exagération quelque peu racoleuse, une telle baisse a assurément de quoi nourrir les interrogations dans une Europe où l'inflation carcérale apparaît comme une tendance sinon générale, du moins dominante ${ }^{3}$.

2 Aussi soudaine qu'inattendue, la diminution récente de la population pénitentiaire allemande prend à contre-pied la plupart des observateurs, dont les auteurs de ces lignes. En dépit de l'érection déjà ancienne de l'Allemagne au rang de « seul grand État européen [qui] échappe au mouvement général de durcissement carcéral $»^{4}$ grâce aux garanties liées à l'ancrage d'un État social fortement inclusif, miser sur l'hypothèse d'une telle baisse était risqué tant cette caractérisation flatteuse de la République fédérale, en même temps qu'elle semble validée par l'évolution récente, dégage paradoxalement un double et doux parfum d'anachronisme. D'abord au titre du décalage, au moment même où elle 
était formulée. En 2003-2004 l'effectif carcéral allemand atteignait en fait un point maximum, à la suite d'une croissance soutenue depuis la Réunification; c'est l'époque où la question du surencombrement des établissements pénitentiaires bat son plein, le phénomène touchant la quasi-totalité des régions dans les structures dites fermées ${ }^{5}$. Signe parmi d'autres, cette question faisait la couverture de la revue spécialisée Neue Kriminalpolitik fin 2003, tandis que des auteurs s'interrogeaient avec plus ou moins de réussite sur les facteurs de la hausse carcérale ${ }^{6}$. Avec le recul d'une décennie, la même question a été abordée en termes de caducité. En effet, l'étendue et la solidité des protections "offertes" - les guillemets s'imposent tant le mot est mal choisi pour désigner le fruit de conquêtes sociales - par le Sozialstaat allemand se sont notablement effritées depuis ${ }^{7}$. Et, parallèlement, on soutiendra difficilement qu'au cours de cette période l'évolution de la législation pénale a brillé par son assouplissement ${ }^{8}$. La difficulté qu'éprouvent les chercheurs de l'université de Greifswald qui suivent depuis plusieurs décennies l'évolution de la démographie carcérale dans leur pays à expliquer la tendance actuelle $e^{9}$ est un bon indice du caractère inattendu, sinon énigmatique de la baisse notable du nombre de détenus en Allemagne.

Essentiellement descriptive, cette contribution ne prétend pas livrer une explication digne de ce nom : il faudrait pour cela conduire une recherche approfondie qui croiserait enquête qualitative et étude quantitative, sachant que les statistiques publiques officielles en la matière sont parfois d'un maniement délicat et accusent certaines lacunes ${ }^{10}$, outre le fait que, on le verra, les données peuvent varier pour les mêmes années selon les sources en fonction des conventions et des critères retenus. Elle espère néanmoins, en première approche, fournir des éléments utiles de mise en perspective et proposer quelques pistes à suivre en s'appuyant presque exclusivement sur des sources disponibles en ligne auxquelles chacun(e) pourra se référer directement pour en approfondir la lecture.

\section{Physionomie d'un retournement de tendance}

Revenons sur la baisse carcérale en question. L'article de Slate sur lequel nous nous sommes fondés et ceux de Die Welt et de la Süddeutsche Zeitung sur lesquels il repose ne font pas cavaliers seuls. Outre plusieurs communiqués de presse de l'agence statistique fédérale ${ }^{11}$, de nombreuses coupures de presse allemande témoignent d'une baisse significative de la population pénitentiaire, à la suite notamment de la publication à l'été 2013 de chiffres officiels concernant l'année 2012 ${ }^{12}$. La tendance démographique y est parfois abordée sous un angle faussement anecdotique, qui en dit long sur la place de la prison dans l'espace public et l'évolution des représentations sociales en la matière. À cet égard, le meilleur exemple est l'article du Spiegel paru en 2013, qui fait part de la vente par certaines régions de prisons désaffectées et bien situées à des investisseurs privés qui les rénovent pour en faire des hôtels, des appartements de standing ou des lieux artistiques. Il donne l'exemple d'un homme d'affaires ayant acheté $37000 €$ l'ancienne prison de Stendal au Land de Saxe-Anhalt avant d'investir plus de deux millions d'euros dans sa conversion en une trentaine d'appartements. Au sud-ouest du pays, deux hommes d'affaires ont entrepris de consacrer cinq millions d'euros à transformer l'ancienne prison d'Offenburg en hôtel, avec cette fois le souhait affiché de conserver délibérément son cachet d'origine. D'autres affaires juteuses sont en perspective, puisque d'autres prisons ont fermé ou doivent fermer dans le pays - en Basse-Saxe, en Saxe-Anhalt, à Berlin ou encore dans le Bade-Wurtemberg ${ }^{13}$. 
5 Du côté des médias français, le démographe Pierre-Victor Tournier avait attiré l'attention, fin 2011, sur la baisse de l'effectif carcéral outre-Rhin sur le site du Nouvel Observateur. À partir des données de Statistique pénale annuelle du Conseil de l'Europe (SPACE), il signalait qu'entre 2001 et 2009 l'Allemagne connaissait un processus de déflation avec une baisse frôlant les $7 \%$, son taux de détention passant pour sa part de 96 à 89 , pendant qu'en France le nombre de détenus grossissait de $31 \%$ avec un taux de détention bondissant de 77 à 96 pour 100000 habitants $^{14}$.

6 Voilà des tendances qui offrent un vif contraste avec ce que l'on pouvait lire il y a encore quelques années. La tonalité était alors à la déploration de records à la hausse et le thème de la surpopulation paraissait bien ancré15. Dans un exposé des tendances pénales publié en 2008, Axel Boetticher et Johannes Feest mentionnaient ainsi la baisse, alors toute récente, du taux de détention entre 2004 et 2007, mais cette mention restait anecdotique dans un tableau d'ensemble qui laissait plutôt voir un durcissement sécuritaire a priori propice à la croissance carcérale ${ }^{16}$. Même le Zweiter Periodischer Sicherheitsbericht, élaboré sous l'égide du ministère fédéral de la Justice, mettait en avant la hausse tendancielle du nombre de détenus, le renforcement de la pénalisation et la sur-occupation partielle des prisons qui en résultait ${ }^{17}$.

7 Aussi bienvenu soit-il, le retournement de tendance récent incite cependant à la vigilance. Et invite finalement à la nuance. D’abord, le phénomène national de diminution de la population carcérale s'accompagne d'importantes disparités régionales. Dans l'article de Slate, l'exemple de la Basse-Saxe, où le nombre de détenus ne cesse de diminuer depuis 2004, est pris comme révélateur d'une situation générale, sans qu'il soit réellement typique. Structurellement, il s'agit d'une région pour ainsi dire septentrionale traditionnellement moins répressive que les Länder du sud. Conjoncturellement, elle est de surcroît dirigée depuis février 2013 par une coalition de gouvernement de gauche au sein de laquelle les Verts, historiquement libéraux en matière pénale, ont pourvu le poste de ministre de la Justice, une situation actuellement unique à l'échelle du pays. Le site du International Centre for Prison Studies (ICPS), affilié à l'université d'Essex, confirme la particularité du Nord-Ouest de l'Allemagne en publiant les taux de détention régionaux, qui oscillent entre 40 pour 100000 dans le Schleswig-Holstein, région rurale frontalière du Danemark, et 113 à Berlin, seul cas où le nombre dépasse 100, en passant par 90 en Bavière $^{18}$. On retrouve approximativement ces ordres de grandeur si l'on se réfère à l'annuaire statistique de 2013 (les chiffres datant de 2011) ${ }^{19}$. Ces inégalités territoriales ne démentent certes pas la logique d'ensemble - une stagnation du nombre de détenus dans la première moitié des années 2000 suivie d'une baisse sensible dans la seconde - mais, contrairement à ce que semble suggérer l'article de Slate, elles ne sont pas négligeables.

Ensuite, celui-ci met sur la piste d'une explication peu convaincante : « une baisse de la criminalité, et en particulier du nombre d'homicides ", elle-même résultant, selon un argument prêté à Christian Pfeiffer (professeur d'université, juriste, directeur de l'Institut de criminologie de Basse-Saxe et ancien ministre de la Justice SPD de la région au début des années 2000) qu'on retrouve formulé ailleurs de manière un peu plus étoffée, du vieillissement démographique du pays. Cette évolution se répercuterait non seulement sur le volume, mais aussi sur les types d'infractions commises ou du moins enregistrées, le retrait des catégories d'infractions passibles de prison étant au principe de la baisse du nombre de détenus (le vieillissement de l'âge moyen intra-muros ayant de surcroît pour avantage un effet pacificateur facilitant la gestion de la détention) ${ }^{20}$. Si elle a le mérite de ne pas souffler l'idée d'une clémence accrue de la justice exposant celle-ci à la critique 
conservatrice bien connue sur le thème du laxisme, l'explication par la baisse du nombre d'homicides peine à convaincre eu égard à la part très faible voire infime du nombre de ces derniers dans le total des infractions condamnées et a fortiori enregistrées. Si l'on s'en tient à la statistique policière (donc sans prendre en considération les possibles requalifications ultérieures), un exposé chiffré paru en 2009 à partir de données de 2007 indiquait ainsi la rareté des homicides : pour 100000 habitants, quatre homicides, contre plus de 3000 vols ou encore quelque 300 infractions à la législation sur les stupéfiants ${ }^{21}$. En 2011, sur près de 6 millions d'infractions comptabilisées par la statistique policière, la catégorie «Mord und Totschlag ", qui inclut les tentatives, n'enregistrait pas plus de 2174 occurrences ${ }^{22}$.

En outre, l'article français, certes court, laisse de côté plusieurs aspects abordés dans ses sources allemandes ${ }^{23}$. Ainsi de l'opposition «massive » de la CDU et du FDP, ainsi que des personnels pénitentiaires, à la politique carcérale de la ministre écologiste de Basse-Saxe. Ainsi aussi du projet, porté «depuis plus de vingt-cinq ans» par des juristes universitaires, d'une nouvelle législation fédérale qui se concentrerait moins sur l'aménagement de la vie carcérale que sur l'après-prison. En dépit de son titre, "Beaucoup de cellules vides en prison », la tonalité de l'article de la Süddeutsche Zeitung, signé par l'un de ces universitaires réformistes, est critique. Le texte signale les multiples travers et lacunes d'une organisation judiciaire complexe ${ }^{24}$ et se fait l'écho des plaidoyers pour une réorganisation en faveur du milieu ouvert davantage que d'une amélioration pénitentiaire. D'ailleurs, on peut entendre des avis divergents concernant la situation actuelle, invitant d'autant plus à éprouver la validité d'une décrue carcérale si massive et générale qu'elle rendrait les prisons caduques. Ainsi dans une émission de radio suisse diffusée en mars 2014, qui rendait compte d'un projet d'hébergement de détenus suisses en surnombre dans des prisons du sud de l'Allemagne, réputées délaissées. À la question : "les prisons allemandes sont-elles à moitié vides? ", la réponse était, à partir des cas de la Bavière et du Bade-Wurtemberg : non, en fait, elles sont presque pleines ${ }^{25}$.

Enfin, l'article de Slate commente la nette diminution du nombre de personnes détenues " au cours de ces dernières années " sans la rapporter à la très forte augmentation caractéristique de la période précédente, de la Réunification au premier tiers des années 2000 (tableau 1).

Tableau 1. - Évolution du nombre de détenus et du taux de détention depuis 1992

\begin{tabular}{|l|l|l|}
\hline Année & Nombre de détenus & Taux de détention \\
\hline 1992 & 57448 & 71 \\
\hline 1995 & 66146 & 81 \\
\hline 1998 & 78592 & 96 \\
\hline 2001 & 80333 & 98 \\
\hline 2004 & 81166 & 98 \\
\hline 2007 & 75719 & 92 \\
\hline 2010 & 72052 & 88 \\
\hline
\end{tabular}




\begin{tabular}{|l|l|l|}
\hline 2013 & 65710 & 81 \\
\hline
\end{tabular}

Source : International Centre for prison Studies ("Germany"). L'ICPS reprend les données de la statistique publique allemande (date de référence au 31 mars).

11 Resserrons à présent l'observation sur le moment du basculement, cette fois à partir de la base de données SPACE déjà mentionnée : on constate alors que c'est après avoir frôlé la barre des 80000 détenus en 2003-2004 que le pays passe sous la barre des 70000 moins d'une décennie plus tard, les années 2004-2006 représentant une période de tassement transitionnelle avant la baisse réelle et continue des années suivantes (tableau 2).

Tableau 2. - Évolution du nombre de détenus et du taux de détention (2002-2012).

\begin{tabular}{|l|l|l|}
\hline Année & Nombre de détenus & Taux de détention \\
\hline 2002 & 78506 & 95,2 \\
\hline 2003 & 79567 & 96,4 \\
\hline 2004 & 79676 & 96,5 \\
\hline 2005 & 78992 & 95,7 \\
\hline 2006 & 79146 & 95,8 \\
\hline 2007 & 77868 & 94,5 \\
\hline 2008 & 74706 & 90,7 \\
\hline 2009 & 73263 & 89,3 \\
\hline 2010 & 71634 & 87,6 \\
\hline 2011 & 70931 & 86,8 \\
\hline 2012 & 69268 & 84,6 \\
\hline
\end{tabular}

Source : Conseil de l'Europe, SPACE I, rapports annuels. La date de référence pour la République fédérale est le 31 mars (contrairement à la norme générale fixée par SPACE au $7^{\text {er }}$ septembre). 2013 n'est pas indiquée car les données ne seront disponibles qu'au 30 novembre. Les données de 2001 sont exclues car la date de référence est aussi indiquée à cette même date. Pour 2004, le rapport n'indique exceptionnellement pas que la date allemande de référence est le 31 mars au lieu du $7^{\text {er }}$ septembre, mais il s'agit vraisemblablement d'un oubli.

12 Si l'on prend les extremums (2004 et 2012), la baisse est d'un peu plus de 13\%. Si l'on se place maintenant à l'échelle de la décennie, on constate qu'entre 2002 et 2012 la baisse est de moins de $12 \%$. Celle-ci n'a certes rien de négligeable, surtout au vu de la situation en France, mais rien à voir non plus avec les $22 \%$ évoqués dans l'article de Slate à partir de chiffres tirés de la Süddeutsche Zeitung. Il faut au passage remarquer ici que l'article français commet une erreur, qui fausse la comparaison établie entre la France et l'Allemagne. Les chiffres de l'article allemand sur lequel il s'appuie ne sont pas ceux des personnes «incarcérées » en général, comme il y est écrit, mais ceux des personnes 
condamnées (Menschen in Strafhaft oder Verwahrung), à l'exclusion donc des personnes placées en détention provisoire (Untersuchungshaft). Ceci invite à distinguer entre les condamnés et les prévenus ; nous y reviendrons.

Malgré ces nuances nécessaires à la compréhension du phénomène dans sa complexité, la baisse est suffisante pour attirer des regards étrangers, américains et britanniques plutôt que français ${ }^{26}$. Les États-Unis et le Royaume-Uni symbolisent à divers degrés une dérive carcérale qui ne règle en rien les questions dites, de façon réductrice, "de sécurité »" Cette préoccupation s'est traduite récemment par la production de rapports spécifiquement dédiés à cette question.

\section{La situation allemande au rapport}

Sentencing and Prison Practices in Germany and the Netherlands: Implications for the United States: le titre du rapport publié en octobre 2013 par le Vera Institute et le Prison Law Office, relayé notamment par un éditorial du New York Times ${ }^{28}$, est assez clair quant à ses motivations. Ce rapport mérite d'être analysé au second degré : son intérêt tient moins à son contenu, souvent attendu et parfois problématique (l'une des figures compare des chiffres de 2010 avec d'autres de 2004), qu'à ce que sa simple existence révèle. Celle-ci est autant, voire davantage, symptomatique de ce qui se passe aux États-Unis - étant donné l'impasse de son système carcéral hypertrophié, de son taux d'incarcération extravagant (près de dix fois plus élevé que celui de l'Allemagne) - que de la situation allemande. On peut d'ailleurs l'inscrire dans un intérêt récurrent du point de vue états-unien. «Pourquoi donc les politiques pénales allemandes ne sont-elles pas plus dures et le taux d'emprisonnement plus élevé ?» se demandait Michael Tonry en 2004 au vu de la stabilité relative (toute relative, on l'a vu, d'autant que l'article parait au moment du pic) du taux d'incarcération en RFA depuis les années $1970^{29}$. Dans ce texte dont le champ d'étude excède en fait largement la République fédérale, il mettait en relief plusieurs facteurs (parmi lesquels une culture politique portée au consensus, l'indépendance des juges, une certaine stabilité des politiques publiques profitant notamment du fait que le savoir universitaire exerce une certaine influence sur les décideurs, etc.) par opposition au fonctionnement états-unien. Le rapport du Vera Institute, pour sa part, évoque en préambule des signes de changement récents aux États-Unis, où l'ampleur des coûts entraînés par le système carcéral suscite une volonté grandissante de le dégonfler.

Ce rapport résulte de la visite, en février 2013, de délégations de trois États fédérés (Colorado, Géorgie, sans oublier la Pennsylvanie, d'ailleurs berceau du modèle pénitentiaire du même nom) en Allemagne et aux Pays-Bas (qu'on laisse ici de côté), d'une semaine dans chaque pays. On peut regretter de ne trouver dans le texte la trace de ces visites en chair et en os que dans quelques très brèves citations. Quoi qu'il en soit, le rapport est porté par une question explicite : comment faire pour confirmer la décrue qui s'amorce aux États-Unis, quelles idées et recettes de réforme peut-on importer? Car du point de vue des observateurs, le cas allemand est exotique à tout point de vue ou presque : volonté affichée de réduire l'emprise de la prison au profit d'autres formes de sanctions, importance proclamée de la resocialisation et de la réhabilitation, efforts pour aménager des conditions de détention qui ne soient pas trop avilissantes, existence d'activités éducatives et sociales ou encore aménagements de peine à l'extérieur ${ }^{30}$. Le rapport souligne notamment l'étonnement des délégués devant le fait que même pour une partie des infractions considérées comme sérieuses, correspondant en tout cas à ce 
que le droit états-unien considère comme des crimes (felonies), on retrouve la volonté de limiter la voie carcérale au profit des amendes, des travaux d'intérêt général (TIG) ou du sursis assorti ou non de mise à l'épreuve. Les délégués sont, d'après le rapport, repartis avec non seulement des idées mais des projets d'application pratiques pour leurs États respectifs.

Ce rapport n'est pas isolé : il fait suite à un autre, publié en mai 2012 pour le compte de la Criminal Justice Alliance, et dont le titre et le sous-titre (Reducing the use of imprisonment. What can we learn from Europe?) sont encore plus explicites quant à la volonté de tirer des leçons d'expériences étrangères ${ }^{31}$. Le regard est cette fois britannique : le rapport a été rédigé par Rob Allen, présenté comme un chercheur indépendant, co-fondateur du site Justice \& prisons et ancien directeur (2005-2010) du International Centre for Prison Studies précité. Il est expert critique du suremploi de la prison, notamment pour les mineurs. Ce n'est alors pas sans étonnement que l'on découvre que le rapport débute par un avant-propos rédigé par une ancienne plume de David Cameron, l'éditorialiste Ian Birrell, critiquant l'état du débat public et les politiques étatiques menées, parlant notamment d'un élan répressif caractérisé de "politics of the madhouse ». Ce rapport, antérieur à celui du Vera Institute et cité par ce dernier, est aussi plus consistant; le champ géographique couvert est également plus large.

Le rapport situe en 2004 le point de rupture inaugurant des divergences entre pays européens, avec d'un côté la République fédérale et les Pays-Bas, de l'autre le RoyaumeUni (en Angleterre et en Écosse la population carcérale a presque doublé en vingt ans) ainsi que la France. De là, il met en relief plusieurs aspects - rapidement encastrés dans des éléments plus généraux (importance de l'État social, culture politique portée à la modération, etc.) - dont on retient ici les principaux, sans reprendre des éléments déjà évoqués. Rob Allen attire tout particulièrement l'attention sur les mesures dites de "diversion» au niveau du parquet (p. 8), autrement dit les alternatives aux poursuites. Des classements sous conditions (sanction pécuniaire, TIG, médiations) peuvent être effectués pour certains délits (Vergehen, par opposition à Verbrechen, les crimes, même si la délimitation et la répartition exactes diffèrent de celle qui a cours en France), incluant la plupart des atteintes aux biens, mais aussi des atteintes à l'intégrité physique (les procédures de médiations concernent des faits de violence dans environ la moitié des cas). Ce filtrage s'accompagne d'une ventilation au moment du jugement: à la "diversion" s'ajoute la "bifurcation", qui tend à réserver la prison pour certaines catégories (auteurs d'infractions sexuelles par exemple) et à l'éviter pour d'autres. L'auteur insiste sur le fait que les magistrats allemands recourent fréquemment au sursis (p.10). Autre élément saillant du point de vue britannique: le fait qu'une certaine souplesse est de mise à l'égard des probationnaires s'ils ne remplissent pas totalement ou strictement leurs obligations, d'où une baisse de la part des infracteurs réincarcérés pour non respect de ces obligations, les choses étant nettement plus strictes au Royaume-Uni. Il signale aussi la contribution non négligeable des universitaires et des groupes confessionnels (à la fois auprès des décideurs et des médias) dans la jugulation du nombre de détenus. Conformément à l'une des leçons principales de la sociologie pénale, il n'y a pas de lien automatique et direct, ni même parfois décelable, entre le niveau de la criminalité et le niveau d'emprisonnement. La baisse de la criminalité enregistrée depuis le milieu des années 2000, après une période de stabilisation, ne suffit nullement à expliquer la décrue carcérale. Ceci d'autant que cette baisse recouvre des tendances 
distinctes, avec notamment une hausse des infractions impliquant des faits de violence, et à l'inverse une baisse des infractions visant les biens sans violence.

De façon générale, la lecture de ces rapports suscite une curieuse impression de déjà vu. Cette diminution rappelle un précédent tout aussi inopiné : celui qui eut lieu entre 1983 et 1990. Déjà alors, la question de l'« exemple » allemand se posait.

\section{Du précédent des années 1980 à la tendance récente}

Ce n'est pas la première fois que l'Allemagne fait, du moins momentanément, figure de modèle présumé aux yeux de certains observateurs, quitte à ce qu'ils déchantent après plus ample examen. "L'Allemagne, un modèle à suivre pour la Grande-Bretagne?", s'interrogeait ainsi un trio d'universitaires en 1992 avant de donner une réponse pour le moins mitigée et globalement négative ${ }^{32}$. Ce qui justifiait leur question était la baisse de la population carcérale à hauteur d'environ un quart de son effectif au cours de la période 1983-1990; une baisse inattendue dans le contexte du virage néoconservateur pris au niveau fédéral sous la férule de la CDU. Le premier article cherchant à expliquer les raisons de la baisse du nombre de détenus en République fédérale au cours des années 1980 était d'ailleurs l'œuvre d'un auteur rattaché au Home Office ${ }^{33}$.

On ne peut ici revenir en détail sur cet épisode sur lequel, à la suite de textes étrangers, André Kuhn s'était penché au milieu des années 1990, mettant en relief un changement de pratiques de la part des magistrats, en particulier à l'égard des jeunes, et un moindre recours à l'emprisonnement pour un certain nombre d'infractions, ressortissant en particulier aux atteintes aux biens ${ }^{34}$. La Réunification avait opposé un brutal coup d'arrêt à cette tendance déclinante et les années 1990 ont ensuite été marquées par une inflation carcérale soutenue. Mais d'une certaine manière, tout se passe un peu comme si la République fédérale reprenait actuellement une tendance à la baisse inscrite dans la moyenne et la longue durée historique (dans les années 1960, la part des peines de prison ferme se situait entre $25 \%$ et $30 \%$, contre moins de $10 \%$ dans les années 2000 ), mais interrompue par les effets de l'absorption/reconstruction du système hérité de l'ancienne RDA. Ainsi, l'Allemagne revenait à l'esprit des réformes de la législation pénale des années 1960 qui avaient préparé le terrain à la loi pénitentiaire fédérale de 1976 - une loi paradoxalement caduque, qui ne reste en vigueur que par défaut dans les quelques régions qui n'ont pas encore édicté leur propre texte ${ }^{35}$ - en faisant de la prison un ultime recours. L'essentiel est alors, sur la longue durée historique encore une fois, un déplacement de la pénalité qui se traduit notamment dans une part des peines pécuniaires tournant aujourd'hui autour des trois quarts. À cet égard, les modalités de la baisse actuelle semblent très voisines de celles qu'elles furent trente ans plus tôt.

En première approche, les effets de plusieurs facteurs se cumulent. Il y a d'abord le fait que la baisse concerne tant les condamnés que les prévenus (tableau 3). La temporalité toutefois diffère. Quand, dans un rapport publié en 2009 (à partir de statistiques de 2007), un début de retournement de tendance est évoqué, c'est la baisse de la détention provisoire qui est mise en avant ${ }^{36}$. Elle a en effet débuté dès le milieu des années 1990 . On est passé d'environ 21000 prévenus en 1995 à 11500 en 2009, soit une baisse qui s'approche de la moitié de l'effectif de départ en quinze ans, et se retrouve autant, voire plus, à l'Est qu'à l'Ouest du pays. 
Tableau 3. - Évolution des condamnés et des prévenus depuis 1995

\begin{tabular}{|l|l|l|}
\hline Année & Condamnés & Prévenus \\
\hline 1995 & 46516 & 20959 \\
\hline 2000 & 60798 & 18322 \\
\hline 2007 & 64700 & 12357 \\
\hline 2008 & 62348 & 11577 \\
\hline 2009 & 61878 & 11138 \\
\hline 2010 & 60693 & 10781 \\
\hline 2011 & 60067 & 10793 \\
\hline 2012 & 58073 & 10982 \\
\hline 2013 & 56662 & 11271 \\
\hline
\end{tabular}

Sources : Statistisches Bundesamt, Strafvollzug, Fachserie 10 Reihe 4.1, 2013 (pour les condamnés) ; Justiz auf einen Blick, 2011 (pour les prévenus en 1995 et 2000) ; Bestand der Gefangenen und Verwahrten in den deutschen Justizvollzugsanstalten, novembre 2013 (pour les autres dates) ${ }^{37}$. Les condamnés incluent les prisonniers sous le régime de la Sicherungsverwahrung. Dates de référence : 31 mars pour les condamnés et 30 novembre pour les prévenus.

Mais outre le moindre recours à la détention provisoire, la statistique publique témoigne d'un phénomène plus récent qui y ajoute ses effets: l'effritement régulier des condamnations. Elles concernaient 897000 personnes (Verurteile) en 2007, 874690 en 2008, 844520 en 2009, 813270 en 2010, 807800 en 2011 et enfin 773900 en 2012, soit une baisse d'environ $14 \%$ sur la période $2007-2012^{38}$.

D'après le communiqué de presse de février 2014 accompagnant la publication des chiffres les plus récents, communiqué qui signale au passage que le plus gros poste statistique est constitué des infractions au code de la route (22\%), les peines de prison (mineurs inclus) représentent $18 \%$ des sanctions, et environ $70 \%$ des peines de prison sont assorties d'un sursis, ramenant la part des peines de prison ferme à environ 6-7\% (41 000 sur 636000 en 2011 ; la proportion était proche de $9 \%$ en 2003). S'il y a bien une surreprésentation des plus jeunes (Heranwachsende, jusqu'à 20 ans inclus) dans les statistiques judiciaires par rapport à leur importance dans la population générale, l'agence fédérale enregistre une baisse considérable de $26 \%$ des condamnations pour les individus de moins de 21 ans entre 2007 et 2012. À cela s'ajoute le fait que l'indicateur de la durée moyenne de détention fourni par SPACE, disponible à partir de 2006, parait luimême témoigner d'une stagnation à la baisse sur la période la plus récente : 8 mois en 2006, 8,3 en 2007 et 8,5 en 2008, puis à nouveau 8 mois en 2009, 7,4 mois en 2010 et 7,6 mois en 2011 (contre environ 8,7 en France au même moment). Il s'agit certes d'une tendance hésitante et fragile qui reste à confirmer, mais le simple fait que cette durée moyenne n'augmente pas est en soi déjà significatif. 

données chiffrées relatives aux condamnés et aux prévenus, mais aussi celles relatives à une autre forme d'enfermement, de type médico-légal, distinguée non seulement dans le code pénal mais aussi dans les statistiques officielles ${ }^{41}$. À côté des statistiques relatives à l'exécution des peines proprement dite (Strafvollzug) existent en effet celles relatives à ce que la catégorisation officielle nomme Maßregelvollzug, soit la détention judiciairement prononcée en raison de troubles psychiatriques ou à des fins de désintoxication. Les individus détenus sous ce régime d'internement thérapeutique, classé dans le code pénal parmi les mesures de sûretée ${ }^{42}$, le sont dans des unités hospitalières sécurisées; ils ne dépendent pas de l'administration pénitentiaire, mais des ministères régionaux chargés de la santé. En prenant en compte ce régime de détention particulier comptabilisé à part (la catégorisation statistique ne recouvrant pas la catégorisation juridique), la baisse dont il a été question jusqu'ici devient alors sensiblement moins franche. dernières décennies - au moins dans la partie occidentale du pays puisque, on l'a dit, une statistique agrégée manque toujours au niveau fédéral (de sorte que l'organisme prend parfois pour certaines régions des données de l'année précédente). Leur nombre est passé de 3649 à l'époque de la Réunification en 1990 à 10471 en 2013. Considérer, comme précédemment, la période 2002-2012 donne à voir une augmentation continue (tableau 4). 
Tableau 4. - Effectif des détenus à des fins de traitement psychiatrique et assimilé ( Maßregelvollzug), 2002-2012

\begin{tabular}{|l|l|}
\hline Année & Effectif \\
\hline 2002 & 6550 \\
\hline 2003 & 7399 \\
\hline 2004 & 7802 \\
\hline 2005 & 8113 \\
\hline 2006 & 8536 \\
\hline 2007 & 8664 \\
\hline 2008 & 8943 \\
\hline 2009 & 9251 \\
\hline 2010 & 9590 \\
\hline 2011 & 9974 \\
\hline 2012 & 10276 \\
\hline
\end{tabular}

Source : Statistisches Bundesamt, Im psychiatrischen Krankenhaus und in der Entziehungsanstalt aufgrund strafrichterlicher Anordnung Untergebrachte (Maßregelvollzug), 2012-2013, avril 2014. Anciens Länder uniquement.

Si cette hausse d'environ $57 \%$ sur une décennie ne suffit pas à compenser la baisse dont il a été question auparavant, les effectifs ne sont nullement négligeables, avec une augmentation de près de 2500 personnes depuis 2004. En Rhénanie-du-Nord-Westphalie par exemple, la région la plus peuplée du pays, 3000 personnes étaient l'année dernière incarcérées à ce titre, contre seulement 1800 personnes dix ans plus tôt ${ }^{43}$. Autrement dit, la question classique du phénomène de vases communicants - ou plutôt de mélange des genres - entre asile et prison n'a pas épuisé sa pertinence.

Tenir compte non seulement des fluctuations quantitatives, mais aussi des changements qualitatifs à l'œuvre, suppose d'ailleurs d'être attentif à la recrudescence de la rétention de sûreté (Sicherungsverwahrung). Alors que le nombre de détenus sous ce régime (comptabilisés dans la statistique principale) restait sous la barre des 200 entre 1985 et 1995, il approchait les 500 en 2009-2010. Favorisée par l'assouplissement graduel des conditions de placement en rétention de sûreté entre 1998 et $2008^{44}$, cette tendance à la hausse semble avoir résisté aux récentes condamnations du dispositif par la Cour européenne des droits de l'homme (CEDH) et la Cour constitutionnelle de Karlsruhe ${ }^{45}$ ainsi qu'aux ajustements législatifs qui s'ensuivirent.

Parallèlement, obtenir une vue d'ensemble requiert de prendre en considération la détention des étrangers en séjour irrégulier avant expulsion (Abschiebungshaft), dont une partie de plus en plus réduite a lieu dans des Justizvollzuganstalten (JVA) et est par 
conséquent comptabilisée dans la statistique des Gefangenen und Verwahrten, contrairement à ceux, beaucoup plus nombreux, incarcérés dans des centres de rétention qui dépendent généralement des ministères de l'Intérieur régionaux. En l'occurrence, les chiffres sont dans les deux cas à la baisse. Du côté des JVA, les données officielles indiquent que 346 personnes étaient détenues sous ce régime en 2013 contre 1786 dix ans plus tôt ${ }^{46}$. Quant à la totalité de la population concernée elle est passée, d'après un rapport récent de l'association Pro Asyl, de 8800 personnes en 2008 à moins de 6500 en 2011 , en raison notamment de changements juridiques effectués en 2009. On constate là encore des disparités régionales fortes (de $-60 \%$ à Hambourg à $+46 \%$ en Saxe-Anhalt en quatre ans). De plus, si les chiffres absolus baissent, la proportion de personnes retenues effectivement "éloignées » reste stable ( $40 \%$ - ce chiffre est comparable aux données disponibles pour la France ces dernières années $)^{47}$.

Il n'est pas non plus inutile de se pencher sur les tendances de l'équivalent fonctionnel de notre dit «milieu ouvert ». La base de données SPACE II, qui concerne les mesures de probation et s'appuie, sauf exception, sur la statistique publique allemande, fournit les chiffres suivants pour les toutes dernières années uniquement : 148805 probationnaires en 2009, 156861 en 2010, 156440 en 2011 et 156358 en 2012, autrement dit une poussée entre 2009 et 2010 (de plus de 5\%) suivie d'une stabilisation. Précisons que les années précédentes étaient caractérisées par une hausse importante: le Zweiter Periodischer Sicherheitsbericht précité indique le chiffre d'environ 135000 probationnaires en $2002^{48}$. Tournons-nous à nouveau vers l'agence statistique fédérale, quoique là encore, il n'existe toujours pas de données agrégées au niveau fédéral; celles disponibles excluent les nouveaux Länder mais aussi Hambourg, et à l'inverse incluent Berlin ${ }^{49}$. Il s'agit donc d'un indicateur partiel et approximatif, tout de même instructif. Sur la longue durée, c'est-àdire depuis la collecte de cette donnée en 1963, la tendance est clairement celle d'une hausse continue. Il importe cependant de différencier le cas des mineurs de celui des adultes $^{50}$. Alors qu'en 1975 on comptait environ 61500 personnes soumises à mesure de probation, la barre des 100000 personnes a été franchie dans la première moitié des années 1980, puis celle des 150000 à l'orée des années 2000. C'est là, comme en France, la grande mutation de la pénalité contemporaine. Complétant le point de vue, les données qui suivent (tableau 5) ne concernent plus seulement les individus soumis à une mesure de probation, mais le nombre de mesures de probation en cours d'exécution à la fin de chaque année civile ; elles prennent en compte le fait qu'une personne condamnée pour plusieurs infractions dans des procédures distinctes peut être simultanément soumise à plusieurs contrôles.

Tableau 5. - Nombre de mesures de probation en cours d'exécution (2001-2011)

\begin{tabular}{|l|l|}
\hline Année & Effectif \\
\hline 2001 & 156951 \\
\hline 2002 & 161211 \\
\hline 2003 & 167216 \\
\hline 2004 & 168704 \\
\hline
\end{tabular}




\begin{tabular}{|l|l|}
\hline 2005 & 171058 \\
\hline 2006 & 175020 \\
\hline 2007 & 177353 \\
\hline 2008 & 182736 \\
\hline 2009 & 182240 \\
\hline 2010 & 180074 \\
\hline 2011 & 182715 \\
\hline
\end{tabular}

Source : Statistisches Bundesamt, Bewährungshilfe, Fachserie 10, Reihe 5, 2013 (données 2011). Date de référence : 31 décembre.

Si la hausse parait plus ou moins jugulée, en 2008-2011, elle est en revanche importante depuis le pic carcéral de 2003-2004 et au-delà. En d'autres termes, si le nombre de détenus baisse, le nombre de personnes sous main de justice et le nombre de mesures de probation, eux, augmente. C'est aussi à cette aune qu'il faut envisager la question évoquée plus haut du "tournant punitif ", surtout dans le contexte d'une baisse de la criminalité enregistrée. En outre, d'autres critères, y compris quantitatifs, méritent d'être pris en compte dans une telle caractérisation, comme le nombre d'aménagements de peine à l'extérieur, ces derniers ayant subi de sévères restrictions dans certaines régions dans les années $2000^{51}$. Autrement dit, plutôt qu'une opposition convenue entre «milieu ouvert » et «milieu fermé », c'est leur complémentarité fonctionnelle (et d'ailleurs symbolique) qui mérite de retenir l'attention. À ce titre, et comme c'est classiquement le cas dans l'histoire de la pénalité, on a pu deviner que le facteur économique, quoique visible parfois seulement en filigrane, était déterminant. Fermer des prisons, comme en BasseSaxe, coûte moins cher que mettre en œuvre des programmes de rénovation ${ }^{52}$.

Par ailleurs, si on conçoit aisément que la décompression carcérale rend moins difficiles les interactions en détention, tant du point de vue des prisonniers que de celui des personnels qui y travaillent, on ne peut oublier que la situation carcérale reste tout à fait problématique et la question carcérale bien vivace, bien que rarement rendue visible. Les associations actives sur ce terrain ne cessent de le signaler et divers articles de presse insistent sur non seulement le quasi-abandon des pratiques mais aussi du thème même de la resocialisation, consacré juridiquement dans les années 1970, sans parler de la pauvreté des moyens qui lui sont alloués ${ }^{53}$. L'illustration la plus emblématique de la période récente est sans doute l'étude menée par l'universitaire Frank Neubacher entre 2010 et 2013, étude relayée médiatiquement en raison de son ampleur (plus de 6000 répondants à un questionnaire distribué dans plus de trente établissements répartis sur cinq régions), pour le meilleur et pour le pire (le retour du thème de la « sous-culture» de la violence). Elle a montré, s'il était besoin (car c'est tout sauf une nouveauté : plusieurs affaires de torture ont défrayé la chronique ces dernières années), que la violence traverse les rapports quotidiens en détention, notamment chez les jeunes, où la majorité en font l'expérience, comme auteur et/ou comme victime ${ }^{54}$. De quoi relativiser l'idée d'une gestion carcérale tendanciellement plus facile qu'auparavant, idée qui va d'ailleurs à rebours de la grande majorité des discours professionnels et publics depuis vingt ans, 
lesquels accentuent au contraire les difficultés résultant des transformations de la composition sociologique des prisonniers du fait, notamment, de réserver la prison aux crimes et délits considérés les plus graves. Un journal modéré comme Die Zeit est depuis des années critique envers la situation carcérale, dont il dresse régulièrement des constats accablants $\mathrm{s}^{55}$, dans des termes qui ne varient que très marginalement par rapport à l'histoire des critiques adressées à la prison ${ }^{56}$.

\section{En conclusion : rouvrir la question carcérale}

On ne peut que se réjouir de la décrue carcérale observable en Allemagne, surtout si elle s'inscrit dans un processus de longue durée, et plus encore si cette érosion est susceptible de reposer plus généralement, dans le débat public, la question de l'existence même de cette institution. La question du fonctionnement (interne) de la prison ne doit toutefois pas masquer celle de ses fonctions (sociales). Si un état des lieux quantitatif et qualitatif de la situation carcérale et, plus largement, pénitentiaire, peut assurément être utile, signalons en conclusion qu'il ne saurait cependant tenir lieu d'analyse sociologique de la question carcérale. L'héritage commun des sciences sociales, par-delà des différences d'approche parfois considérables, consiste à référer la prison à autre chose qu'à elle-même : à l'état du marché du travail, aux inégalités socio-économiques, aux antagonismes de classe, à l'iniquité des procédures judiciaires, au fonctionnement des institutions d'encadrement des classes populaires, aux conditions d'exercice de la souveraineté étatique, etc. En d'autres termes, découpler la question carcérale de la question sociale la vide de son sens. Le rappel s'impose en Allemagne plus encore qu'en France et le mélange de juridisme et de sociologie platement descriptive qui imprègne la majorité des travaux sur le sujet contribue à en nourrir une représentation tronquée et carcéro-centrée. Même lorsque l'accent est mis sur la dureté des conditions de détention et les affres de la condition recluse, même quand elles entendent résister au réductionnisme sécuritaire et se prononcent en faveur d'un ajustement humanitaire à un seuil de dignité présumé convenable, les formulations dominantes du problème, avant tout morales et non sociopolitiques, contribuent à leur manière à rétrécir, sinon à refermer la question carcérale. Adossées à une représentation individualisante des rapports sociaux qui aplanit les rapports de domination structurant le monde social, elles passent sous silence le rôle pivot de la prison dans ce que Michel Foucault appelait la « gestion différentielle des illégalismes ». Celle-là même qui, en particulier, assure un traitement de faveur autant symbolique que judiciaire à la "délinquance en col blanc", quand bien même « tout indique que la corruption est intégrée aux mœurs des entreprises outre-Rhin (...) tellement ancrée dans la culture commerciale que ce comportement apparait souvent comme une donnée structurelle», ce dont le cas de Peter Hartz fournit un condensé édifiant ${ }^{57}$. 


\section{NOTES}

1. Annabelle Georgen, «En Allemagne, on ferme des prisons faute de détenus ", Slate.fr, 23/01/2014. Précisons que cet article est une version entièrement revue et augmentée d'un texte paru initialement sur le site "Délinquance, justice et autres questions de société ", animé par Laurent Mucchielli. Outre ce dernier, nous remercions Emmanuel Blanchard, Fabien Jobard et Camille Lancelevée pour leurs remarques sur la version initiale.

2. Mathilde Schnee, «Allemagne : la fin des prisons », ARTE Journal, 03/03/2014.

3. Le même site semble d'ailleurs s'être fait une spécialité de détecter les situations atypiques: voir Camille Jourdan, «Aux Pays-Bas, il y a plus de gardiens de prison que de prisonniers. Mais c'est une exception... », Slate.fr, 14/04/2014.

4. Hugues Lagrange, Demandes de sécurité. France, Europe, États-Unis, Paris : Seuil/République des idées, 2003, p. 7. En novembre 2013, c'est la Suède qui faisait l'objet d'un traitement médiatique écrit et audiovisuel ; du côté de la presse, voir par exemple « La Suède ferme des prisons faute de détenus », Le Figaro, 12/11/2013 ; «Faute de détenus, Suède et Pays-Bas ferment des prisons », Le Parisien, 12/11/2013 ; "En Suède, les prisons mettent la clé sous la porte ", M, le Magazine du Monde, 22/11/2013 ; "Pourquoi la Suède ferme ses prisons ", Le Point, 30/11/2013. Cet intérêt pour une «bonne élève » traditionnelle reste toutefois moins surprenant que la mise en avant subite de l'Allemagne.

5. Frieder Dünkel/Bernd Geng/Christine Morgenstern, «Strafvollzug in Deutschland. Aktuelle rechtstatsächliche Befunde », Forum Strafvollzug, n 59, 2010, p. 20 et p. 29.

6. «Überbelegung im Strafvollzug», Neue Kriminalpolitik, $\mathrm{n}^{\circ} 4$; Stefan Sühling, "Factors Contributing to Rising Imprisonment Figures in Germany ", The Howard Journal of Criminal Justice, $n^{\circ} 1,2003$, p. 55-68.

7. Voir les analyses de l'économiste Arnaud Lechevalier depuis cette date, notamment via son blog. Dans un texte de 2005, il caractérisait le plan « Agenda 2010 » de « cure d'amaigrissement la plus radicale depuis qu'existe la République fédérale ». Id., "Le crépuscule de l'État social allemand ", Alternatives économiques, $\mathrm{n}^{\circ} 234,2005$, p. 39.

8. Expression révélatrice : réagissant au durcissement de la loi sur la rétention de sûreté, le président de la Cour constitutionnelle de Karlsruhe, Klaus Tolksdorf, dénonçait ainsi en 2010 «l'hystérie sécuritaire » à l'œuvre en Allemagne.

9. Frieder Dünkel/Bernd Geng/Christine Morgenstern, "Strafvollzug in Deutschland », art. cit., p. 30. Cette perplexité est relevée par Rob Allen, Reducing the Use of Imprisonment. What Can We Learn from Europe?, Report for the Criminal Justice Alliance, mai 2012, p. 14.

10. Les statistiques de poursuites pénales sont unifiées au niveau fédéral depuis 2007 seulement, il n'existe pas de statistiques de probation agrégées au niveau fédéral en raison des lacunes dans plusieurs nouveaux Länder, etc. Pour un relevé détaillé des biais et lacunes des statistiques officielles, voir Wolfgang Heinz, «Probleme des Systems der Kriminal- und Rechtspflegestatistiken und ihre bundesgesetzlichen Lösungsmöglichkeiten », Konstanzer Inventar Sanktionsforschung (KIS), mai 2012. Le site du KIS offre plus généralement de nombreuses ressources, notamment sur la délinquance juvénile. Pour une analyse critique, à partir d'un autre domaine, des effets de la pauvreté sociologique de la statistique publique allemande, voir Andreas Pfeuffer/Franz Schultheis, "Quelques particularités allemandes dans la représentation statistique du monde social », Sociétés contemporaines, $\mathrm{n}^{\circ}$ 45-46, 2002, p. 17-42.

11. Dernier en date: Statistisches Bundesamt, «Zahl der Verurteilten im Jahr 2012 weiter rückläufig », Pressemitteilung vom 12. Februar 2014, n 46, 2014. 
12. Par exemple Matthias Reiche, "Seit Jahren geht die Zahl der Strafgefangenen zurück", Mdr.de, 09/08/2013, à partir des cas (distincts) de la Saxe-Anhalt et de la Thuringe. L'article se termine en signalant qu'en Saxe, contrairement aux deux Länder précités, la population carcérale augmente à nouveau depuis 2009, d'où une situation de surnombre.

13. Stephan Degenhardt, « Zu Hause in der Zelle », Der Spiegel, n 49, décembre 2013, p. 50.

14. Pierre-Victor Tournier, « Prisons : quand on a le choix, mieux vaut être détenu en Allemagne qu'en France », tempsreel.nouvelobs.com, 07/12/2011.

15. Voir par exemple «Zahl der Strafgefangenen auf Rekordniveau », Tagesspiegel.de, 15/02/2007.

16. Axel Boetticher/Johannes Feest, « German Criminal and Prison Policy », in : Peter Johan Paul Tak/Manon Jendly (dir.), Prison Policy and Prisoners' Rights, Nijmegen : Wolf Legal Publishers, 2008, p.361-390. Comme souvent dans la littérature académique nationale, cette vue d'ensemble instructive pâtit d'un prisme juridique.

17. BMJ, Zweiter Sicherheitsbericht (2. PSB), 2006, p. 577 et p. 606 notamment. Ce rapport, le deuxième du nom (le précédent date de 2001), n'a curieusement pas connu de successeur.

18. Voir le site de l'ICPS, qui reprend des données de la statistique publique allemande.

19. Les taux de détention s'étalent alors entre 48 (Schleswig-Holstein, suivi par le Brandebourg avec 55) et 124 (Berlin, suivi par la Bavière avec 96) pour une moyenne nationale de 83 (Statistisches Bundesamt, Statistisches Jahrbuch, 2013, p. 297). Notons ici que le taux de détention est calculé à partir d'un effectif qui comprend les personnes détenues par rétention de sûreté ( Sicherungsverwahrung) et celles en attente d'expulsion du territoire (Abschiebungshaft), deux catégories qui, à la fin des années 2000 , contribuaient chacune pour environ $1 \%$ de l'ensemble.

20. "Warum sich die Gefängnisse leeren », RP-Online.de, 08/08/2013. On y lit en même temps que, contrairement à une idée reçue, la délinquance juvénile baisse aussi ces dernières années. On verra plus loin qu'en tout cas, le nombre de condamnations baisse depuis 2007 pour les moins de 21 ans.

21. Jörg-Martin Jehle, Criminal Justice in Germany. Facts and Figures, Berlin : Federal Ministry of Justice, 2009, p. 10-11.

22. Statistisches Jahrbuch 2013, op. cit., p. 303.

23. "Land schließt Gefängnisse in Celle und Braunschweig", Welt.de, 22/01/2014 ; Heribert Prantl, «Im Knast sind viele Zellen frei », Sueddeutsche.de, 23/01/2014.

24. La réforme du fédéralisme de 2006 a redistribué aux régions le pouvoir législatif en matière pénitentiaire : voir Grégory Salle, « De l'éclatement juridique à la stratification sociale. Actualité de la question carcérale en Allemagne », Cultures et Sociétés, n 10, 2009, p. 46-51.

25. Émission « Factuel », RTS.ch, 24 mars 2014.

26. Le rapport d'information déposé en conclusion des travaux d'une mission d'information sur les moyens de lutte contre la surpopulation carcérale le 23 janvier 2013 par les députés de la majorité et de l'opposition Dominique Raimbourg (PS) et Sébastien Huyghe (UMP) ne mentionne ainsi pas le cas allemand.

27. On trouve même des témoignages de prisonniers états-uniens et anglais qui affirment préférer purger leur peine en RFA plutôt que dans leurs pays respectifs. Voir Fred Searle, «Brits and Americans choose German jails ", Thelocal.de, 22/11/2013.

28. «Lessons from European Prisons », The New York Times, 07/11/2013.

29. Michael Tonry, «Why Aren't German Penal Policies Harsher and Imprisonment Rates Higher?», German Law Journal, n5, 2004, p. 1187-1206.

30. Concernant cet écart, voir le livre de James Whitman, Harsh Justice. Criminal Punishment and the Widening Divide between America and Europe, New York : Oxford University Press, 2003.

31. Rob Allen, Reducing the Use of Imprisonment, op. cit. La Criminal Justice Alliance se présente comme une coalition de 67 organisations (philanthropiques, universitaires, syndicales, associatives, etc.) impliquées dans les politiques et les pratiques pénales. 
32. Renate Prowse/Hartmut-Michael Weber/Chas Wilson, «Rights and Prisons in Germany: Blueprint for Britain? », International Journal of the Sociology of Law, $n^{\circ} 20,1992$, p. 111-134.

33. John Graham, «Decarceration in the Federal Republic of Germany: How Practitioners Are Succeeding Where Policy-Makers Have Failed », British Journal of Criminology, n², 1990, p. 150-170. Voir aussi Johannes Feest, «Reducing the prison population: Lessons from the West German experience? », John Muncie/Richard Sparks (dir.), Imprisonment: European Perspectives, New York : St Martin's Press, 1991, p. 131-145.

34. André Kuhn, "Étude des fluctuations de la population carcérale allemande ", Déviance et société, $n^{\circ} 1,1996$, p. 59-83. Notons que le périmètre de son étude était restreint aux anciens Länder. L'auteur évoquait déjà, à titre d'hypothèse, le facteur de la dénatalité et la régression de la part des classes d'âge jeunes (p. 70), mais en lui accordant un rôle a priori minime et nécessitant en tout cas de retracer les médiations le reliant aux fluctuations de la population carcérale.

35. Voir Grégory Salle, «Petite histoire de la loi pénitentiaire allemande », Le Monde diplomatique, $\mathrm{n}^{\circ}$ 672, mars 2010, p. 4-5.

36. Jörg-Martin Jehle, Criminal Justice in Germany, op. cit., p. 45-48.

37. Notons que même à la même date de référence, en l'espèce le 30 novembre, les chiffres peuvent différer légèrement, selon notamment qu'ils prennent en compte ou non les personnes temporairement à l'extérieur.

38. Sources : Statistisches Bundesamt, «Zahl der Verurteilten im Jahr 2012 weiter rückläufig », art. cit. ; Statistisches Jahrbuch 2013, op. cit., p. 304. Chiffres arrondis (soit dans les sources, soit par les auteurs).

39. Un bon exemple est le panorama effectué par Dietrich Oberwittler/Sven Höfer, « Crime and Justice in Germany. An Analysis of Recent Trends and Research », European Journal of Criminology, $\mathrm{n}^{\circ}$ 4, 2005, p. 465-508.

40. Frieder Dünkel/Bernd Geng/Christine Morgenstern, «Strafvollzug in Deutschland », art. cit., p. 31.

41. Pour une vue d'ensemble du système judiciaire (et notamment pénal) allemand à partir des statistiques officielles, voir la brochure du Statistisches Bundesamt Justiz auf einen Blick. Ausgabe 2011, février 2011 (la date de référence sur le plan pénitentiaire est, ici, le 30 novembre).

42. Sur le régime juridique de ces mesures et les « dérives » récentes de la Sicherungsverwahrung, voir Xavier Pin, «L'internement de sûreté en Allemagne : une mesure de défense sociale à la dérive ", Déviance et Société, $n^{\circ} 4,2010$, p. 527-545.

43. « 3000 psychisch kranke Straftäter sitzen im Maßregelvollzug », RP.Online, 08/08/2013.

44. Voir par exemple Tillmann Bartsch, «Aspekte der Sicherungsverwahrung in Straf- und Maßregelvollzug ", in: Britta Bannenberg/Jörg-Martin Jehle (dir.), Gewaltdelinquenz. Lange Freiheitsentziehung. Delinquenzverläufe, 2011, p. 291-309.

45. CEDH, arrêt du 17 décembre 2009, Affaire Mücke contre Allemagne ; CEDH, arrêt du 13 janvier 2011, Affaire Haidn contre Allemagne ; Cour constitutionnelle de Karlsruhe, arrêt du 4 mai 2011.

46. Bestand der Gefangenen und Verwahrten, op. cit., juillet 2014, p. 18 et p. 168.

47. Marei Pelzer/Uli Sextro, Schutzlos hinter Gittern. Abschiebungshaft in Deutschland, Frankfurt/M. : Pro Asyl, 2013, p. 50. Un tiers des mises en détention sont jugées inconstitutionnelles (Marei Pelzer, « Abschiebungshaft : Totale Institution in der Legitimationskrise », Tag des Flüchtlings 2013, Frankfurt/M. : Pro Asyl, 2013, p. 43).

48. 2. PSB, op. cit., p. 596.

49. Statistisches Bundesamt, Bewährungshilfe, $\mathrm{n}^{\circ}$ 5, 2013 (données 2011).

50. Sur la longue durée la hausse est, pour les adultes, à peu près permanente depuis la Réunification, voire depuis 1963. Pour les mineurs la courbe est différente, montrant une baisse sensible entamée avant la moitié des années 1980 et poursuivie jusqu'au début des années 1990, puis à nouveau un tassement voire une baisse ces dernières années. 
51. Frieder Dünkel/Bernd Geng/Christine Morgenstern, «Strafvollzug in Deutschland », art. cit., p. 30.

52. Cette idée est développée par Teresa Havlicek, «Neuer Trend im Strafvollzug. Rot-Grün schleift Knäste ", taz.de, 22/01/2014.

53. Voir par exemple Heribert Prantl, "Im Keller der Gesellschaft », Sueddeutsche Zeitung, 20/11/2008.

54. Voir par exemple Martin Kotynek/Stephan Lebert/Daniel Müller, «Gewalt ist in deutschen Gefängnissen Alltag », ZeitOnline, 15/08/2012 ; Benjamin Schulz, « Gefängnisalltag in Deutschland. Weggesperrt und vergessen ", Spiegel.Online, 15/01/2013.

55. Voir par exemple, à dix ans d'intervalle, Martin Klingst, « Einsperren ist teuer und sinnlos ", Zeit.Online, 25/04/2002 ; id., "Die Schlechterungsanstalt », Zeit.Online, 22/08/2012. Dans un long entretien au quotidien, Bernd Maelicke, ancien responsable des prisons de la région du Schleswig-Holstein (présentées comme un modèle de réussite eu égard à leur taux de détention très bas) pendant quinze ans, évalue à environ la moitié le nombre de détenus qui ne devraient pas être en prison : « Zur Verbrechen gemacht », Zeit.Online, 06/03/2014.

56. Pour un aperçu de la question carcérale en RFA dans les années 1950-1970, saisie à travers son traitement par le Spiegel, voir Grégory Salle, «La question carcérale en République fédérale d'Allemagne au reflet du Spiegel (1947-1979) », Criminocorpus, revue hypermédia, Varia, septembre 2013.

57. Jean Guisnel, Armes de corruption massive, Paris : La Découverte, 2011, chap. 5. Rappelons que Peter Hartz, condamné à deux ans de prison avec sursis en 2007 pour une affaire de corruption au sein de son entreprise Volkswagen, a été, en tant que conseiller du chancelier Gerhard Schröder (et annoncé récemment comme possible conseiller de l'Élysée après une visite au chef de l'État français), l'artisan des « réformes » qui, au nom de la «souplesse » du marché du travail, ont défait les protections sociales et institutionnalisé la pauvreté laborieuse contrainte, soit la marginalisation dont la prison est un outil de gestion.

\section{AUTEURS}

\section{GRÉGORY SALLE}

(chargé de recherche au CNRS, rattaché au CLERSÉ, Lille)

\section{BARBARA BAUDUIN}

(doctorante au Centre Marc Bloch, Berlin et au laboratoire PACTE, Grenoble) 